05

\title{
Внутреннее трение на границах зерен, содержащих протяженные поры
}

\author{
() В.Г. Кульков, А.А. Сыщиков
}

Филиал НИУ „Московский энергетический институт“, Волжский, Волгоградская обл., Россия

E-mail: vikulkov@yandex.ru

Поступило в Редакцию 7 ноября 2018 г.

Предлагается модель внутреннего трения на межзеренной границе, содержащей взаимно равноудаленные параллельные цилиндрические поры. Под действием переменного сдвигового напряжения происходит взаимное смещение приграничных областей, сопрягаемых на сегментах между порами в зависимости от их расположения. Найдены величина рассеянной энергии на каждом сегменте и суммарное внутреннее трение. Температурная зависимость внутреннего трения имеет вид широкого пика.

DOI: 10.21883/PJTF.2019.03.47267.17580

Влияние пор на физические свойства твердого тела весьма разнообразно. Чувствительными к наличию пор являются плотность материала, его теплопроводность, предел прочности, коррозионная стойкость и др. [1,2]. В литературе рассматривается применение металлических пористых материалов в областях защиты окружающей среды, энергетики, химической промышленности и медицины [3]. Конфигурации пор в металлах различны: от равноосных до длинных вытянутых в одном направлении цилиндрических [4]. Исследованию внутреннего трения в пористых материалах посвящено довольно много работ. Это относится как к объемным образцам [5,6], так и к пленкам [7]. В настоящей работе рассматривается механизм зернограничного внутреннего трения с участием цилиндрических пор, расположенных параллельно друг другу и лежащих в межзеренных границах.

Рассмотрим участок границы, ограниченный двумя тройными стыками зерен и содержащий $2 N+2$ параллельных одинаковых цилиндрических пор радиуса $a$ (см. рисунок, $a$ ). Крайние из них расположены вдоль тройных стыков. Пусть вдоль границы действует постоянное сдвиговое напряжение $\sigma^{0}$. С учетом симметрии относительно каждого из двух сопрягающихся зерен можно рассмотреть деформацию только одного из них. Для нахождения деформации граничных точек зерна будем считать, что к свободной от соседнего зерна поверхности приложена равномерно распределенная по сегментам сдвиговая сила $\sigma^{0} l$ на единицу длины вдоль образующей пор (см. рисунок, $b$ ). Полагаем, что эта сила приводит к однородному смещению сегмента поверхности между соседними порами как целого. Смещение соседних сегментов различно. При этом взаимное смещение берегов разрезанной цилиндрической поры в виде протяженной ложбинки имеет упругий характер с жесткостью $\alpha$, что схематически изображено пружинками на рисунке, $c$. Нумерация сегментов для $N=2$ показана на рисунке, $b$. Условия равновесия сегментов приводят к системе уравнений

$$
\begin{cases}\sigma^{0} l-\alpha\left(u_{n}^{0}-u_{n-1}^{0}\right)+\alpha\left(u_{n+1}^{0}-u_{n}^{0}\right)=0, & |n|<N, \\ \sigma^{0} l-2 \alpha u_{n}^{0}+\alpha u_{n+1}^{0}=0, & n=-N, \\ \sigma^{0} l-2 \alpha u_{n}^{0}+\alpha u_{n-1}^{0}=0, & n=N,\end{cases}
$$

где индекс $n(-N<n<N)$ нумерует сегменты. Решением системы (1) являются функции

$$
u_{n}^{0}=\frac{\sigma^{0} l}{2 \alpha}\left((N+1)^{2}-n^{2}\right) .
$$

Взаимное смещение берегов разреза на каждом сегменте границы в 2 раза больше, чем даваемое выражением (2).

Теперь рассмотрим случай переменного сдвигового напряжения, приложенного к границе: $\sigma=\sigma_{0} \exp (i \omega t)$. Переменная во времени деформация отстает по фазе от приложенного напряжения

$$
u_{n}=u_{n 0} \exp \left(i \omega t-\varphi_{n}\right) .
$$

где $u_{n 0}$ и $\varphi_{n}-$ амплитуда и сдвиг фазы на $n$-м сегменте. Для скорости межзеренного проскальзывания можно записать выражение, аналогичное приведенному в работе [8]:

$$
v_{n}=I\left(\sigma_{0} \exp (i \omega t)-\sigma_{n}^{i}\right)
$$

Здесь $v_{n}$ - скорость проскальзывания, $I$ - обратная вязкость границы, $\sigma_{n}^{i}$ - обратное напряжение на сегменте, создаваемое остальными сегментами. В скобках в (4) стоит полное напряжение, действующее на сегменте, являющееся суперпозицией внешнего приложенного к границе напряжения и внутреннего. Выражение для последнего легко получить из (2), где для смещения используется (3). С учетом замечания, следующего после этого выражения, получаем

$$
\sigma_{n}^{i}=\frac{\alpha u_{n}^{0}}{l}\left((N+1)^{2}-n^{2}\right)^{-1} \exp \left(i \omega t-\varphi_{n}\right) .
$$



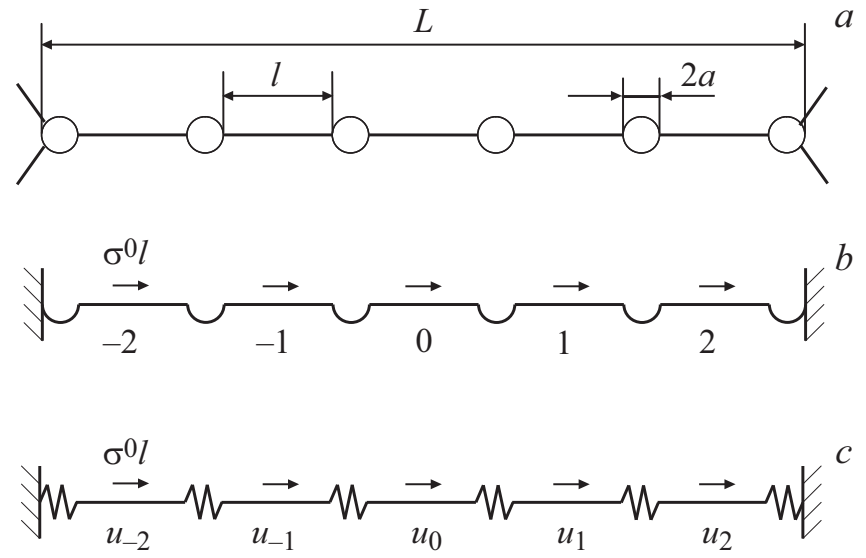

Сечение границы: исходная структура $(a)$, одно из сопрягаемых зерен $(b)$ и замена пор упругими элементами $(c)$.

Подставляя (5) и производную по времени от (3) в (4) и решая полученное уравнение, находим

$$
\begin{aligned}
\operatorname{tg} \varphi_{n}=\omega \tau_{n}, u_{n}^{0} & =\frac{\sigma_{0} l}{\alpha \sqrt{1+\left(\omega \tau_{n}\right)^{2}}}\left((N+1)^{2}-n^{2}\right), \\
\tau_{n} & =\frac{l}{\alpha I}\left((N+1)^{2}-n^{2}\right) .
\end{aligned}
$$

Рассеянную на сегменте за период колебаний энергию найдем как [8]:

$$
\Delta W_{n}=l \int_{0}^{\pi / \omega} \operatorname{Re} \sigma \cdot \operatorname{Re} v_{n} d t
$$

Здесь Re - действительная часть комплексной величины. Внутреннее трение, обусловленное вкладом $n$-го сегмента, равно $Q_{n}^{-1}=\frac{\Delta W_{n}}{W}$, где $W=\frac{V \sigma_{0}^{2}}{2 G}-$ упругая энергия, запасенная в зерне, $V-$ его объем, $G-$ модуль сдвига.

Внутреннее трение на всем участке границы $Q^{-1}$ между тройными стыками зерен равно сумме выражений для каждого сегмента. Воспользовавшись (3), (6) и (7), находим

$$
Q^{-1}=\frac{\pi l^{2} G}{\alpha \beta V} \sum_{n=-N}^{N} \frac{\omega \tau_{n}}{1+\left(\omega \tau_{n}\right)^{2}}\left((N+1)^{2}-n^{2}\right),
$$

где $\beta$ - коэффициент формы зерна, показывающий долю принадлежности объема зерна к площади рассматриваемого участка границы между тройными стыками.

Коэффициент жесткости $\alpha$ можно оценить следующим образом. По обе стороны от ложбинки смещения соседних с ней сегментов одного из зерен различаются на $\xi_{n}=u_{n+1}-u_{n}$. Деформация в окрестности ложбинки подобна деформации вокруг краевой дислокации с модулем вектора Бюргерса $\xi_{n}$. Полная сила на сегменте определяется интегрированием напряжения в плоскости скольжения от дислокации по размеру сегмента и делением на 2, поскольку рассматривается только полупространство по одну сторону от плоскости скольжения дислокации. Вклад второго полупространства отсутствует.

$$
F_{n}=\frac{1}{2} \int_{a}^{l+a} \frac{G \xi_{n}}{2 \pi(1-\varepsilon)} \frac{d x}{x}
$$

где $\varepsilon-$ коэффициент Пуассона, $x-$ координата вдоль сегмента по нормали к образующей поры. Взяв интеграл в (9), получим коэффициент жесткости, связывающий силу с относительным смещением сегментов: $\alpha=G / 4 \pi(1-\varepsilon) \ln ((l+a) / a)$. Окончательное выражение для внутреннего трения принимает вид

$$
Q^{-1}=\frac{4 \pi^{2} l^{2}(1-\varepsilon)}{\beta V \ln ((l+a) / a)} \sum_{n=-N}^{N} \frac{\omega \tau_{n}}{1+\left(\omega \tau_{n}\right)^{2}}\left((N+1)^{2}-n^{2}\right) .
$$

Экспериментальные исследования показывают, что ширина зернограничных пиков внутреннего трения всегда больше дебаевской $[9,10]$. Это связано с распределением участков границ по размерам, типу границ, а также с нелинейностью зависимости скорости проскальзывания от напряжения [11]. Согласно (10), еще одним фактором уширения пиков является наличие пор на границе. Как видно из этого выражения, результирующий пик образуется наложением нескольких максимумов с разными временами релаксации различной высоты. Поэтому ширина пика зависит от количества пор $2 N$. Например, в случае четырех внутренних пор времена релаксации в единицах $l /(\alpha I)$ равны 9, 8 и 5; в случае шести внутренних пор - 16, 15, 12 и 7. Высоты накладываемых пиков Дебая в единицах $4 \pi^{2} l^{2}(1-\varepsilon) / \beta V \ln ((l+a) / a)$ равны 9,16 и 10 в первом случае и 16, 30, 24 и 14 во втором. Центр распределения времен релаксации близок по величине ко времени релаксации беспористых границ [8].

Полный спектр внутреннего трения на пористых границах зерен содержит также фон, монотонно возрастающий с температурой. Величина фона может лежать в не зависящей от амплитуды области напряжений [12] либо зависеть от величины приложенных напряжений [13].

\section{Список литературы}

[1] Черемской П.Г., Слезов В.В., Бетехтин В.И. Поры в твердом теле. М.: Энергоатомиздат, 1990. $374 \mathrm{c}$.

[2] Liu P.S., Chen G.F. Porous materials: processing and applications. Butterworth-Heinemann, 2014. $576 \mathrm{p}$.

[3] Qin J., Chen Q., Yang Ch., Huang Y. // J. Alloys Compd. 2016. V. 654. P. 39-44.

[4] Nakajima H. // Prog. Mater. Sci. 2007. V. 52. N 7. P. 10911173.

[5] Golovin I.S., Sinning H.-R. // Mater. Sci. Eng. A. 2004. V. 370. N 1-2. P. 504-511. 
[6] Ota K., Ohashi K., Nakajima H. // Mater. Sci. Eng. A. 2003. V. 341. N 1-2. P. 139-143.

[7] Du G., Tan Z., Li Z., Liu K., Lin Z., Ba Ya., Ba D. // Current Appl. Phys. 2018. V. 18. N 11. P. 1388-1392.

[8] Кульков В.Г. // Изв. вузов. Физика. 2005. Т. 48. № 4. С. 93 94.

[9] David E., Ian J. // Mater. Sci. Eng. A. 2018. V. 730. P. 425437.

[10] Fan G.D., Zheng M.Y., Hu X.S., Xu C., Wu K., Golovin I.S. // J. Alloys Compd. 2013. V. 549. P. 38-45.

[11] Кульков В.Г. // Металлы. 2005. № 4. С. 69-73.

[12] Кульков В.Г., Васильева Ю.В. // Перспективные материалы. 2009. № 7. С. 171-175.

[13] Кульков В.Г., Васильева Ю.В., Дешевых В.В. // Письма в ЖТФ. 2012. Т. 38. В. 23. С. $40-45$. 